Investigación

\title{
PREVALENCIA DEL DETERIORO COGNITIVO EN PERSONAS MAYORES DE 60 AÑOS, EN UN CONSULTORIO MÉDICO DE LA REGIÓN DE ALTAHABANA (CUBA), PERTENECIENTE AL MUNICIPIO BOYEROS. AÑO 2020
}

PREVALENCE OF COGNITIVE IMPAIRMENT IN PEOPLE OVER 60 YEARS OLD AT A MEDICAL OFFICE IN THE REGION OF ALTAHABANA (CUBA), BELONGING TO THE BOYEROS MUNICIPALITY. YEAR 2020

\section{Yudit Hernández Esterlin \\ Licenciada en Enfermería \\ Máster en Longevidad Satisfactoria \\ Profesor Auxiliar, Facultad de Ciencias Médicas Dr. "Enrique Cabrera”, La Habana, Cuba yudithdez@infomed.sld.cu}

\section{Cruz María Contreras Torres}

Licenciada en Enfermería

Máster en Formación Didáctica

Profesor Auxiliar, Facultad de Ciencias Médicas Dr. "Enrique Cabrera”, La Habana, Cuba cardicec@infomed.sld.cu

\section{Gilberto Piedra Ruíz}

Licenciado en Enfermería

Máster en Enfermería

Profesor Auxiliar, Facultad de Ciencias Médicas Dr. "Enrique Cabrera”, La Habana, Cuba gilbertopr@infomed.sld.cu

Artículo recibido el 23 de julio de 2021. Aceptado en versión corregida el 23 de agosto de 2021.

\section{RESUMEN}

El deterioro cognitivo es un problema de salud a nivel mundial, el cual se ha convertido en uno de las mayores demandas de atención médica en las personas mayores. Con el objetivo de determinar la prevalencia del deterioro cognitivo en las personas mayores de 60 años, se realizó un estudio descriptivo, con carácter retrospectivo de corte transversal a 323 adultos mayores en el Consultorio Médico de la Familia \# 20, ubicado en la región de Altahabana, perteneciente al Policlínico Universitario "Federico Capdevila" del Municipio Boyeros (Cuba). El estudio se realizó entre enero y diciembre de 2020. Se encontró que el 31\% de las personas mayores, pertenecen al grupo de edades de 65 a 69 años, con un predominio del género femenino en un $61 \%$. El nivel de escolaridad universitario estuvo presente en un $57,5 \%$. El deterioro cognitivo de las personas mayores prevaleció en un 14\%; la hipertensión 
Prevalencia del deterioro cognitivo en personas mayores de 60 años... arterial, en un $64,8 \%$ fue la enfermedad crónica no transmisible que más se asoció al deterioro cognitivo.

Palabras clave: deterioro cognitivo, persona mayor, enfermedad crónica no transmisible.

\begin{abstract}
Cognitive impairment is a global health problem, which has become one of the greatest demands for medical care in older people. In order to determine the prevalence of cognitive impairment in people over 60 years of age, a descriptive, retrospective, cross-sectional study was carried out on 323 older adults in the Family Medical Clinic \# 20, located in the Altahabana region, belonging to the "Federico Capdevila" University Polyclinic of the Boyeros Municipality (Cuba). The study was carried out between January and December 2020. It was found that $31 \%$ of the elderly belong to the 65 to 69 age group, with a $61 \%$ predominance of the female gender. The level of university education was present in $57.5 \%$. Cognitive impairment in the elderly prevailed in 14\%; arterial hypertension, in $64.8 \%$, was the chronic non-communicable disease that was most associated with cognitive deterioration.
\end{abstract}

Keywords: cognitive impairment, bigger adult, chronic illness not transmissible.

http://dx.doi.org/10.7764/Horiz_Enferm.32.2.118-128

\section{INTRODUCCIÓN}

El envejecimiento progresivo de la población en el siglo XX es un triunfo de la especie humana y refleja logros sin precedentes en la esperanza de vida; esto se debe a una combinación de factores, especialmente la declinación de la mortalidad infantil, disminución de las muertes maternas y los beneficios que surgen del desarrollo socio-económico global: mejor nutrición, disminución de las infecciones, mejor nivel de vida, progreso en educación, atención médica y tecnología biomédica ${ }^{(1)}$.

Este fenómeno constituye un problema a nivel mundial; ejemplo de esto es que en el mundo anualmente la población total crece solamente en $1,7 \%$ y el ritmo de crecimiento de la población mayor de 60 años de edad es de
2,5\%; y a partir del año 2020 crecerá cada vez con mayor intensidad. Entre los años 1990 y 2010 la tasa de crecimiento de la población de la tercera edad permaneció constante, sin embargo, a partir del año 2010, el crecimiento aumentó a 3,1\%; tasas de esta magnitud originarán que la población anciana se duplique cada 23 años. Una de las características relevantes de este proceso en el mundo es la rapidez con que se ha producido, mientras que un país como Francia necesitó casi 200 años para duplicar la población de 60 años y más; en Cuba, este mismo proceso, ocurrió en menos de 40 años ${ }^{(2,3,4)}$.

La población cubana está compuesta por 11.215.344 habitantes, distribuidos en 15 provincias y 168 municipios; el porcentaje de urbanización 
es de un $77,0 \%$ y el $20,4 \%$ de las personas tiene 60 años y más de edad ${ }^{(5)}$.

En este mismo país, el deterioro cognitivo constituye uno de los problemas de salud pública más importantes, dada su relación con la edad, en la última década se presentó un continuo incremento, tanto en su incidencia como en su prevalencia, secundariamente al aumento progresivo de la longevidad en la población ${ }^{(6)}$. Estudios realizados en la población mundial indican que entre un $5 \%$ y un $15 \%$ de la población de más de 65 años, son diagnosticados con demencia. De hecho, constituye, en países desarrollados, la tercera enfermedad en costo social y económico, después de las enfermedades cardíacas y el cáncer, además, ocupa el cuarto lugar en las principales causas de muerte en el $\operatorname{anciano}^{(7)}$.

El presente estudio tiene como objetivo, determinar la prevalencia del deterioro cognitivo de los adultos mayores en el Consultorio Médico de la Familia \# 20, pertenecientes al Policlínico Universitario "Federico Capdevila". Municipio Boyeros (Cuba) durante el 2020. Específicamente se determinó la prevalencia del deterioro cognitivo de los adultos mayores; se identificó el grado de deterioro mental de la muestra estudiada, y se relacionó el deterioro mental y enfermedades crónicas no transmisibles.

\section{MATERIAL Y MÉTODOS}

Se realizó un estudio descriptivo, de carácter retrospectivo, de corte transversal en las personas mayores de 60 años del Consultorio Médico de la Familia \# 20, ubicado en la región de Altahabana, perteneciente al Policlínico Universitario "Federico Capdevila" del Municipio Boyeros. El estudio se realizó entre enero y diciembre de 2020. De un universo de 335 adultos mayores de 60 años y más, se seleccionaron 323 que reunían los siguientes criterios de inclusión: personas mayores de 60 años y más (que estuviesen de acuerdo con participar en la investigación), con residencia permanentemente en el área, que no presentaran afecciones como: imposibilitan la función de los órganos efectores de la sensopercepción (visión y audición), somnolencia, estupor, confusión, delirio, cuadros depresivos profundos, retraso mental severo, esquizofrenia o que se encontraran postrados. Por ello, fueron excluidos 12 del universo total (335). El presente estudio se desarrolló, respetando los aspectos técnicos, éticos y psicológicos que incluye el consentimiento informado.

Se le aplicó el Mini Examen de Estado Mental (Mini-Mental Test Examination, MMSE) (Anexo 1) a toda la muestra, referido a un instrumento que puede ser utilizado para evaluar el estado cognitivo en forma sistemática y exhaustiva de las personas. Específicamente consta de 11 preguntas que analizan áreas de funcionamiento cognitivo: orientación, registro, atención, cálculo, memoria y lenguaje. El resultado del examen se recogió en puntuación cuantitativa. El rango de puntuación fue la siguiente: 1 a 7: deterioro severo de conciencia; 8 a 15: deterioro moderado de conciencia; 16 a 23: deterioro ligero de conciencia; y 24 a 30: deterioro no significativo de la conciencia.

\section{Validez y confiabilidad}

El instrumento MMSE ha sido validado y utilizado extensamente, tanto en la práctica como en la investigación 
Prevalencia del deterioro cognitivo en personas mayores de 60 años...

clínica desde su creación en 1975. En América Latina, el MMSE se modificó y se validó en Chile para encuesta de la OPS Salud, Bienestar y Envejecimiento (SABE), en 1999.

Además del uso de dicho instrumento, se diseñó una encuesta y se le aplicó a toda la muestra, con las siguientes variables: edad, sexo, nivel de escolaridad y tipo de Enfermedad Crónica no Transmisible, cuyos resultados se procesaron en un PC Pentium IV a través del sistema Excel.

Fueron revisadas además las Historias Clínicas de Salud Familiar e Historias Clínicas de salud individual de los adultos mayores de 60 años y más. Se confeccionó una base de datos en una microcomputadora Pentium 4 Microsoft $\mathrm{XP}$, en programa Access, y se procesaron en forma automatizada. Se obtuvieron distribuciones de frecuencia (números y porcentajes) de todas las variables.

\section{RESULTADOS}

La Tabla 1 distribuye a los adultos mayores por grupos de edades y sexo, permitiéndonos conocer que el mayor número de los mismos se encuentran en el grupo etario de 65 a 69 años, donde se encontraron 102 pacientes (31\%); en cuanto al sexo, el femenino con 198 pacientes $(61 \%)$ es superior al sexo masculino con 125 pacientes (39\%).

En la Tabla 2 donde se analiza el nivel de escolaridad de las personas mayores, donde se observa que el 57,58\% (186 personas mayores) tienen nivel universitario; el 21,05\%, nivel técnico medio (68 personas mayores); el 11,45\%, nivel Pre universitario (37 personas); el $7,12 \%$, nivel de secundaria (23 personas); y finalmente, el $2,78 \%$, nivel educacional primaria (9 personas mayores).

Tabla. 1. Distribución de adultos mayores según grupos de edades y sexo. Fuente: Encuesta General.

\begin{tabular}{lllllll}
\hline \multirow{2}{*}{$\begin{array}{l}\text { Grupos } \\
\text { de edades }\end{array}$} & \multicolumn{3}{c}{ Femenino } & \multicolumn{2}{c}{$\begin{array}{l}\text { Género } \\
\text { Masculino }\end{array}$} & \multicolumn{2}{l}{ Total } \\
\cline { 2 - 7 } & No. & $\%$ & No. & $\%$ & No. & $\%$ \\
\hline $60-64$ años & 45 & 23 & 43 & 34 & 88 & 21 \\
$65-69$ años & 65 & 33 & 37 & 30 & 102 & 31 \\
$70-74$ años & 52 & 26 & 30 & 24 & 82 & 25 \\
75 y más & 36 & 18 & 15 & 12 & 51 & 16 \\
Total & 198 & 61 & 125 & 39 & 323 & 100 \\
\hline
\end{tabular}

En la Tabla 3 se analiza la distribución de las personas mayores según deterioro mental, observándose que 278 pacientes
$(86 \%)$ no presentan deterioro mental alguno, mientras que 45 pacientes $(14 \%)$, sí presentan deterioro mental. 
Tabla 2. Distribución de las personas mayores nivel de escolaridad. Fuente. Encuesta general.

\begin{tabular}{lcc}
\hline $\begin{array}{l}\text { Nivel de } \\
\text { escolaridad }\end{array}$ & No. & $\%$ \\
\hline Primaria & 9 & 2,78 \\
Secundaria & 23 & 7,12 \\
Preuniversitaria & 37 & 11,45 \\
Técnico Medio & 68 & 21,05 \\
Universitaria & 186 & 57,58 \\
Total & 323 & 100 \\
\hline
\end{tabular}

Tabla 3. Distribución de las personas mayores según deterioro mental. Fuente: Mini Examen de Estado Mental.

\begin{tabular}{lll}
\hline Deterioro Mental & No. & $\%$ \\
\hline Con Deterioro Mental & 45 & 14 \\
Sin Deterioro Mental & 278 & 86 \\
Total & 323 & 100 \\
\hline
\end{tabular}

En la Tabla 4 se analiza la distribución de las personas mayores, según grado de deterioro mental y género. En ella se puede apreciar que: el deterioro moderado está presente en 21 personas mayores (6\%), el deterioro ligero en 15 personas $(5 \%)$ y con deterioro severo existen 9 personas $(3 \%)$. En cuanto al género, el femenino supera al masculino con 28 pacientes (15\%). Finalmente, 278 pacientes $(86 \%)$ no tienen deterioro mental.

Tabla 4. Distribución de las personas mayores, según grado de deterioro mental y género. Fuente: Mini Examen de Estado Mental.

\begin{tabular}{|c|c|c|c|c|c|c|}
\hline \multirow[b]{3}{*}{$\begin{array}{l}\text { Grado de deterioro } \\
\text { Mental }\end{array}$} & \multicolumn{4}{|c|}{ Género } & \multirow{2}{*}{\multicolumn{2}{|c|}{ Total }} \\
\hline & \multicolumn{2}{|c|}{ Femenino } & \multicolumn{2}{|c|}{ Masculino } & & \\
\hline & No & $\%$ & No & $\%$ & No & $\%$ \\
\hline $\begin{array}{l}\text { Severo } \\
\text { (1-7 puntos) }\end{array}$ & 6 & 3 & 3 & 2 & 9 & 3 \\
\hline $\begin{array}{l}\text { Moderado } \\
\text { (8-15 puntos) }\end{array}$ & 13 & 7 & 8 & 6 & 21 & 6 \\
\hline $\begin{array}{l}\text { Ligero } \\
\text { (16-23 puntos) }\end{array}$ & 9 & 5 & 6 & 5 & 15 & 5 \\
\hline Total & 28 & 15 & 17 & 13 & 45 & 14 \\
\hline
\end{tabular}


Prevalencia del deterioro cognitivo en personas mayores de 60 años...

En la Tabla 5 se muestra la distribución de las personas mayores con Enfermedades Crónicas no Transmisibles, en la cual se puede constatar que la Hipertensión Arterial ocupa el primer lugar con 35 personas mayores $(64,8 \%)$, le continúa la Diabetes mellitus con 18 personas mayores $(30 \%)$, en igualdad de número con las enfermedades cerebrovasculares, mientras que con Cardiopatía Isquémica se encuentran 15 personas $(65,2 \%)$, y, por último, el Asma Bronquial con 5 personas $(62,5 \%)$. Es importante destacar en la Tabla 5 sólo se distribuyen a los adultos mayores que presentan deterioro mental (45 de ellos, que representan un 14\%) y que, además, un paciente puede presentar pluripatologías crónicas. Sin embargo, de ellos, 18 personas (30\%), no presentan enfermedades crónicas no transmisibles.

Tabla 5. Distribución de las personas mayores con Enfermedades

Crónicas no Transmisibles. Fuente: Encuesta General.

\begin{tabular}{lll}
\hline Enfermedades Crónicas no Transmisibles & No. & $\%$ \\
\hline Hipertensión Arterial & 35 & 64,8 \\
Diabetes mellitus & 18 & 30 \\
Cardiopatía Isquémica & 15 & 65,2 \\
Enfermedad Cerebrovascular & 18 & 30 \\
& & \\
Asma Bronquial & 5 & 62,5 \\
Sin Enfermedad Crónica & 18 & 30 \\
\hline
\end{tabular}

\section{DISCUSIÓN}

En el envejecimiento individual ocurre una serie de modificaciones morfológicas, psicológicas, funcionales y bioquímicas que originan el paso del tiempo sobre los seres vivos; donde hay una pérdida progresiva de la capacidad de adaptación y de reserva del organismo ante los cambios, es un proceso dinámico que se inicia en el nacimiento, se desarrolla a lo largo de nuestras vidas, y no es sinónimo de enfermedad ${ }^{(8,9)}$.

La detección temprana del Deterioro Cognitivo resulta de importancia vital, debido a las implicaciones socio individuales, socioeconómicas y familiares que se derivan de la demencia y las dificultades reales de tiempo en su prevención, incluso, en las limitaciones actuales de un tratamiento curativo, por lo que se hace necesario detectar formas iniciales de deterioro cognitivo leve y las enfermedades crónicas que pueden acelerar la evolución o agravar la capacidad cognitiva. Por lo anterior, se plantea que la edad es el factor de riesgo más importante para el declinar $\operatorname{cognitivo}^{(10,11)}$.

De León-Arcila R. et al. (2009), señalan que es la mujer la que predomina con deterioro cognitivo, esto, debido a la presencia de factores socioculturales y además, porque ella es más longeva ${ }^{(12)}$.

Los resultados del presente estudio, en relación al predominio del género femenino con deterioro cognitivo, coinciden con numerosos estudios analizados, los cuales afirman que existe un 
incremento de la prevalencia de Deterioro Cognitivo paralelo a la edad y al género femenino. Los resultados de algunas de estas investigaciones se describen a continuación.

En un estudio realizado por Vega, encontró que el género femenino prevaleció en su muestra, siendo el mayor número de mujeres que sobrepasaban los 65 años de edad, con un Deterioro adquirido $^{(13)}$.

Por otra parte, Fonte Sevillano (2020) obtuvo en su investigación que en las personas mayores de 80 años, un $74,5 \%$ prevaleció con deterioro cognitivo, encontrando significativa influencia en el género femenino ${ }^{(14)}$.

Gómez y Bonnin reportan una frecuencia de deterioro cognitivo de $33 \%$ en el grupo etario de 85 a 89 años de $\operatorname{edad}^{(15)}$.

Estudios realizados por Varona et al., encontraron que el $72,3 \%$ de los pacientes evaluados correspondió al género femenino ${ }^{(16)}$.

En cambio, en países como Colombia y Arabia Saudita se encontró un predominio significativo $(\mathrm{p}<0,05)$ de Deterioro Cognitivo en hombres, comparado con mujeres ${ }^{(17,18)}$.

En cuanto a los resultados del nivel educativo (escolaridad) del presente estudio, su efecto deletéreo pudiera explicarse porque el nivel bajo de escolaridad se asocia frecuentemente a nivel socioeconómico bajo e insuficiencia en nutrición, vivienda y atención a la salud. A su vez, hay que considerar que, en los individuos con niveles más altos de educación, se favorece el desarrollo cerebral, el crecimiento dendrítico y la circulación cerebral, y también ejecutan mejor las pruebas, enmascarando o retrasando el diagnóstico de deterioro $\operatorname{cognitivo}^{(19)}$.

En relación a lo anterior, el Estado cubano determinó que era necesario continuar con la superación de la educación, y asumió la responsabilidad de organizar, controlar y dirigir los procesos docentes que comprendieron la formación, especialización y educación continua de sus recursos humanos, con el objetivo de elevar el nivel económico, la educación, la salud y por consiguiente la calidad de vida de nuestra población ${ }^{(20)}$.

Corona Miranda (2020), plantea en su estudio que la incidencia y el costo de las demencias se incrementan; indica además, que las estrategias de prevención primaria sobre los factores de riesgo pudieran disminuir la carga de esta enfermedad sobre la salud pública ${ }^{(21)}$.

Estudios realizados por Matos Diaz et al. demuestran que las personas mayores con deterioro cognitivo y sus manifestaciones clínicas ligeras, tienen un mayor riesgo de padecer, en un futuro, algún tipo de demencia ${ }^{(22)}$.

Libre Rodriguez, en su estudio epidemiológico realizado en Cuba, en los municipios de Marianao, la Lisa, Playa, Plaza de la Revolución y 10 de Octubre de la Capital y Bauta de la actual provincia de Artemisa, mostró una prevalencia de síndrome demencial entre 8,2 y 11,2\% y específicamente un 5,5\% corresponde a la enfermedad de Alzheimer ${ }^{(23)}$.

En otro estudio cubano, se indica que las personas mayores padecen más enfermedades (pluripatologías), son mucho más vulnerable a todo tipo de agresión, sus mecanismos de defensa son más limitados, en él es menor la reserva 
Prevalencia del deterioro cognitivo en personas mayores de 60 años...

fisiológica, prevalecen las situaciones de discapacidad, consumo elevado de fármacos y necesidad de cuidados continuados. Se ha informado la asociación entre diversas enfermedades crónicas degenerativas (diabetes mellitus, hipertensión, enfermedad cerebrovascular, cardiopatía, osteoartritis, hepatopatías, neoplasias y neurológicas) y la declinación cognitiva y funcional ${ }^{(24)}$.

En el estudio SABE (Salud, Bienestar y Envejecimiento), realizado en Ciudad de La Habana en el 2000-2001, se obtuvo que el $44 \%$ de las personas encuestadas referían Hipertensión arterial, el $14,8 \%$ Diabetes mellitus, el 24,1\% dijo padecer del corazón y el 9,7\% de Enfermedad Cerebro Vascular, el 8,5\% padece de deterioro cognitivo ${ }^{(25)}$.

Valdés King et al., en otro estudio realizado en Cuba, aportaron que del total de su muestra de estudio, el 95\% presentaron enfermedades crónicas no transmisibles como factor de riesgo con deterioro cognitivo ${ }^{(26)}$.

Diferentes autores concuerdan en la presencia de enfermedades crónicas o comorbilidades como factores de riesgo que ocasionan discapacidad funcional: en enfermedades cardiovasculares o pulmonares y la diabetes mellitus tipo 2, considerando los incrementos anuales de incidencia y prevalencia de la diabetes mellitus tipo 2, es entendible el incremento en la discapacidad a causa de sus complicaciones ${ }^{(27,28)}$.

\section{CONCLUSIONES}

En este estudio se determinó que la prevalencia del deterioro cognitivo estuvo presente en menos de la mitad de la muestra estudiada, incidiendo en el grupo de edades de 65 a 69 años, con predominio del género femenino. En cuanto al grado de deterioro cognitivo, el moderado fue el de mayor prevalencia. El mayor nivel educacional fue el universitario y la Hipertensión Arterial fue la enfermedad crónica no transmisible que más se asoció en las personas mayores con deterioro cognitivo.

\section{REFERENCIAS BIBLIOGRÁFICAS}

1. Geriatría. Temas para enfermería. Llanes Betancourt, Caridad. La Habana: Editorial Ciencias Médicas, 2017.434 p.: il., tab.

2. Colectivo de autores. Manual de procedimientos. Enfermería familiar y comunitaria. La Habana: Editorial Ciencias Médicas, 2017.150 p.: il., tab.

3. El envejecimiento y las personas de edad. Indicadores para América y el Caribe. [Separata Unidad]. S.1.: CEPAL.2008.

4. Centenarios en Cuba. Los secretos de la longevidad. La Habana: Publicaciones Acuario,2012. pp. 1-3.

5. Cuba. Oficina Nacional de Estadísticas. El envejecimiento de la población cubana. La Habana. Cuba. Ministerio de Salud Pública. Oficina Nacional de Estadística (ONE) Anuario Estadístico de Salud. La Habana: Minsap; 2020. Disponible en: www.sld.cu/sitios/dne/ o http://bvscuba.sld.cu/anuarioestadistico-de-cuba/

6. Colectivo de autores. Medicina General Integral. Álvarez Sintes R, Br; 3. ed. La Habana: Editorial 
Hernández Esterlin Y, Contreras Torres CM, Piedra Ruíz G

Ciencias Médicas, 2014.358 p., 5 t.: il., tab.

7. Arrieta Antóna P.G., Baz Rodríguez B. Deterioro cognitivo en el paciente con riesgo cardiovascular https://doi.org/10.1016/j.semerg.2021 .01 .009

8. Nolasc, A. Alzheimer. Manual d'instruccions. Madrid: McGraw Hill/Interamericana, 2010. pp. 31-4.

9. Pardo Andreu, G. Consideraciones generales sobre algunas de las teorías del envejecimiento. Madrid: McGraw-Hill/Interamericana, 2011. pp. 67-8.

10. Zamora MM, Castro EF, Avila JA, Manrique BS, Lopez RR, Sosa AL et al. The association between social support and cognitive function in mexican adults aged 50 and older. Arch Gerontol Geriatric. 2017[acceso:20/12/2019];68;113-18.

Disponible en http://www.ncbi.nlm.nih.gov.bvs

11. Rodriguez S, Mora S, Patino A, Garcia G, Escribano H, Garcia O et al. Prevalence of Cognitive impairment in individuals aged over 65 in an urban area; DERIVA STUDY. BMC Neurol.2011[acceso:20/12/2019];11147. Disponible en http://dx.doi.org/10.1186/1471-237711-1479

12. De León-Arcila R. et al. Factores de riesgo para deterioro cognitivo $\mathrm{y}$ funcional en el adulto mayor. Rev Med Inst Mex Seguro Soc 2009; 47 (3): 277-284.

13. Vega A. Prevalencia y factores asociados al Deterioro adquirido. Estudio de caso. Neurología 2016[acceso:20/12/2019];68;113-18.
Disponible

en http://dx.doi.org/10.1016/j.nrl.2016.1 0.002

14. Fonte Sevillano T. Deterioro Cognitivo en personas mayores de 80 años. Revista cubana de medicina. volumen 59 No.1. 2020. Disponible en

revmedicina.sld.cu/index.php/med/art icle/view/314/1147

15. Gómez VN, Bonnin RBM, Gómez MI, Yanez FB, Gonzalez ZA. Caracterización clínica de pacientes con Deterioro Cognitivo. Revista Cubana de Medicina. 2009. [acceso:20/12/2019],42(1):12-

$7 . \quad$ Disponible en http://bvs.Sld.cu/revistas/med/vol.421-03/med 103.htm

16. Varona Gutierrez DG, Cascudo Barre N, Brenes Hernandez L. Deterioro Cognitivo leve y multimorbilidad en el adulto mayor evaluados en la clínica de deterioro cognitivo. CITED. 2014-2016. Revista Geroinfo.Vol.12. No.2.2017 RNPS 2110.

17. Arboleda HE, Aguirre AD, Muñoz C. Pineda AC, Lopera F. Prevalencia de deterioro cognitivo en una población colombiana.RevNeurol.2008[acceso: 20/12/2019],46(12):709-4.Disponible en

http://dialnet.unirioja.es/servlet/revist a

18. Muath A, Abdullah A, Loay B. Prevalence of Mild Cognitive Impairment and Dementia in Saudi Arabia: A community-Based study. Dement Geriatric cogn Disord, 2018[acceso:20/12/2019], Extra (8):98-103.Disponible en 
Prevalencia del deterioro cognitivo en personas mayores de 60 años...

http://www.karger.com/services/open access licence

19. Peterson RC. Mild Cognitive impairent. Continiuum (Minneap Minn). 2016;(22);404-18

20. República de Cuba. Ministerio de Salud Pública. Resolución Ministerial No. 110 Publicado en la Gaceta Oficial de la República, dados en La Ciudad de la Habana en el MINSAP a los 14 días del mes de Agosto del 2004

21. Corona Miranda B, Prado Rodriguez R, Duany Navarro A. Epidemiología de las demencias. Arch Univ Gen Calixto Garcia. 2020;8(2):133147.Acceso:22/08/2021Disponible en http://www..revcalixto.sld.cu/index.p hp/ahag/article/view/514

22. Matos Diaz R, Perez Diaz R, Mendez Amador T. Deterioro Cognitivo Leve y afectividad en el adulto mayor. Rev Hosp Psiq de la Habana.Vol 11 No.2.2014.WWW.revistahph.sld.cu

23. Libre Rodriguez, JJ. Usted puede prevenir la enfermedad de Alzheimer. La Habana: editorial cientificotecnica. 2009. Consultado 22/8/2021.Scielo.Sld.cu/Scielo.php.s cript
24. Roca Goderich, R. et al. Temas de Medicina Interna. 4ta edición.Vol.3. La Habana: Editorial Ciencias Médicas; 2002; 31:211-234.

25. Morfi Samper R. La salud del adulto mayor en el siglo XXI. Rev Cubana Enfermer. 2005;21(3):1. Disponible en:

http://scielo.sld.cu/scielo.php?script= sci_arttext\&pid=S0864-

03192005000300001\&lng=es\&nrm= iso

26. Valdés King M, Gonzalez Caceres JA, Mansur Salisu. Prevalencia de depresión y factores asociados al deterioro cognitivo en el adulto mayor. Revista Cubana Medicina General integral. Volumen 33,No.4.2017

27. Colunga-Rodríguez, Cecilia, García de Alba, Javier E. Caracterización psicosocial de pacientes con diabetes tipo 2 en atención primaria. Rev Cubana Salud Pública v.34 n.4 Ciudad de La Habana oct.-dic. 2008

28. Drurry TF, Danchik KM, Harris MI. Sociodemographic characteristic of adult diabetics. En National Diabetes Data Group. Publication No. 5. 2009. 
Anexo 1. Evaluación Cognitiva (MMSE) Modificada

1. Por favor, dígame la fecha de hoy.

Anote un punto por cada respuesta correcta.

Sondee el mes, el día del mes, el año y el día de la semana.

2. Ahora le voy a nombrar tres objetos. Después que se los diga, le voy a pedir que repita en voz alta los que recuerde, en cualquier orden. Recuerde los objetos porque se los voy a preguntar más adelante.

Lea los nombres de los objetos lentamente y a ritmo constante, aproximadamente una palabra cada dos segundos.

Si para algún objeto, la respuesta no es correcta, repita todos los objetos hasta que el entrevistado se los aprenda (máximo 5 repeticiones). Registre el número de repeticiones que debió

3. Ahora voy a decirle unos números y quiero que me los repita al revés:

$\begin{array}{lllll}1 & 3 & 5 & 7 & 9\end{array}$

Al puntaje máximo de 5 se le reduce uno por cada número que no se mencione, o por cada número que se añada, o por cada número que se mencione fuera del orden indicado.

4. Le voy a dar un papel. Tómelo con su mano derecha, dóblelo por la mitad con ambas manos y colóquelo sobre sus piernas.

Entréguele el papel y anote un punto por cada acción realizada correctamente.

5. Hace un momento le leí una serie de 3 palabras y Ud. repitió las que recordó. Por favor, dígame ahora cuáles recuerda.

Anote un punto por cada objeto recordado.

6. Por favor copie este dibujo:

Muestre al entrevistado el dibujo con los círculos que se cruzan. La acción está correcta si los círculos no se cruzan más de la mitad.

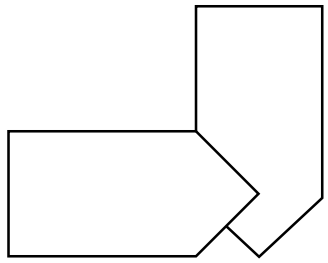

Anote un punto si el objeto está dibujado correcto.

SUME LOS PUNTOS ANOTADOS EN LOS

TOTALES DE LAS PREGUNTAS 1 A 6.

La puntuación máxima es de 19 puntos y a partir de 13 puntos o menos se sugiere déficit cognitivo.

Anote un punto por cada objeto recordado en el primer intento.

Total:

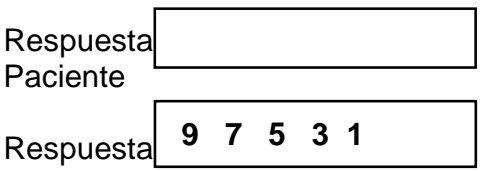
correcta

Total:

Toma papel

Dobla

Coloca

Total:

Árbol

Mesa

Avión

Total:

Correcto:

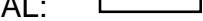

